\title{
Self-discovered breast cancer symptoms and women's help seeking behaviour: key findings from phase one of a two-phase study
}

\author{
Mairin O'Mahony
}

Catherine McAuley School of Nursing \& Midwifery, Brookfield Health

Sciences Complex, UCC

\section{Background}

Breast cancer is the most common cancer among women in the western world. In Ireland, breast cancer was the most common cancer diagnosed amongst women during 2000-2004 with approximately 3,095 cases reported annually and an average of 947 deaths. It is well known that the earlier the diagnosis of breast cancer is made the more likely it is that women will have a better health outcome. However, 20-30\% of women wait for one month or more before presenting to a Health Care Professional with a self discovered breast symptom. This is a worrying situation given the increased emphasis on prompt presentation of symptoms and the associated link with better health care outcomes for women diagnosed with breast cancer. Therefore, more work on help-seeking behaviour from the woman's perspective will help Health Care Professionals to understand women's needs and concerns surrounding symptom discovery and highlight the key issues linked to delayed help seeking. This paper reports on phase one of a two-phase study which explored women's help-seeking behaviour for a self-discovered breast symptom, from a qualitative perspective.

\section{Aim}

The aim of phase one of the study was to explore women's help seeking behaviour for a self-discovered breast symptom, to find out more about women's experience of finding such a symptom and how this influences their help seeking behaviour. In addition, the researcher wanted to identify the key issues to be included in a questionnaire for phase two of the study. 


\section{Methods}

Following ethical approval, a qualitative descriptive study using semi-structured interviews with ten women who had self-discovered a breast symptom was carried out. Initially, women were asked to tell about their experience of finding a breast symptom. Then, more specific areas identified as being important in the literature review were explored with women. These areas included symptom related issues, emotional responses to the symptom, social issues, health seeking habits, access to health services and knowledge and beliefs about the symptom and its outcome. Women's socio-demographic details were recorded at the end of each interview.

\section{Analysis and Results}

The interviews were recorded and transcribed by the researcher, following which all interviews were read and re read several times. Data were then analysed using content analysis, which involved identifying and summarising the key issues emerging for women within each of the main topics outlined above. The researcher was conscious of maintaining the credibility and dependability of the findings throughout the study. This was done by being true to the data, using women's direct quotes to illustrate the points being made, and discussing findings with the co-researchers to see if they agreed with the issues emerging.

\section{Findings}

\section{Socio-demographics, symptom discovery and help seeking behaviour}

Ten women took part in the study ranging in age from 25 to 55 years. These included seven Irish women, two Eastern European women and one English woman. All of the women discovered the symptom(s) themselves. Three women presented with a breast lump, four with breast pain, two with both lump and pain and one with a bloody nipple discharge. Four women had a family history of breast cancer which included aunt for two women and mother and aunt and mother and sister for two women, respectively. The time from symptom discovery to first visiting the General Practitioner (GP) was called "help seeking behaviour" and was either prompt (within one month) or delayed (over one month). Following symptom discovery, six women visited their GP within one month and four delayed over one month. Two of these women delayed from one to two months and two delayed for over three months. 


\section{Factors influencing women's help seeking behaviour}

The key facilitators for women's help-seeking behaviour were telling another person about the symptom; knowing about breast symptoms and their associated risks and the importance of early detection of breast cancer; and confidence in the health services overall. Delayed help-seeking was due mainly to women's denial and fear and family and work commitments. In addition, lack of knowledge in relation to family history and risk and the belief that breast cancer was incurable impacted on delay. The study highlighted that denial impacted on the help seeking behaviour of those women who delayed help seeking for over one month or more, as highlighted by one woman who said:

I thought if I ignored the symptoms, they would go away...

In addition, women experienced varying degrees of fear, causing some to delay and others to seek help earlier. Voicing concerns about the symptom to another person had a positive impact on help-seeking behaviour. In some cases, the competing social roles of family and work commitments prevented women form seeking help at an early stage. Women's knowledge and performance of breast self-examination varied. Notably, some women reported uncertainty around breast self examination and said that they never examined their breasts.

Health service utilisation factors had a positive effect on women's help seeking behaviour and overall, women expressed satisfaction with the GP services. A presenting symptom of a breast lump was significant for most women and caused them to seek help promptly. This supports findings from previous studies which highlight that the nature of the symptom impacts on early help seeking behaviour. Although women were aware that early detection of breast cancer was recommended, this did not always impact positively on their help seeking behaviour. Women believed that family history was the biggest risk factor for developing breast cancer. However, a false sense of security in the absence of a family history of breast cancer was noted amongst some women.

A belief that breast cancer was curable if detected and treated at an early stage was expressed by most women. However, this was not the case for one woman who delayed help seeking for over three months and expressed the pessimistic view that her breast symptom and its outcome would be permanent and incurable. In relation to a breast cancer diagnosis, religious beliefs and having a positive attitude were recognised by most women as being helpful to their health outcome overall. Although religious beliefs were important, few women relied solely on praying to God when they first found their breast symptom. Finally, although a strong belief in the use of conventional medicine for breast cancer treatment was prevalent, women also recognised the value of alternative therapies as an additional treatment. 


\section{Conclusion and recommendations}

This study increases Health Care Professionals' understanding of women's help seeking behaviour on self discovery of a breast symptom and highlights the key issues influencing delayed help seeking. i.e., psychological and social factors and women's knowledge and beliefs. Findings also clarify that these factors have potential to both initiate and inhibit women's help seeking behaviour. Thus, confirming their appropriateness for inclusion in the questionnaire for phase two of the study. The study emphasises the importance of continued focus on the message of early detection of breast cancer. New initiatives that address the barriers to prompt help-seeking need to be developed. In this regard, nurses have an important role to play in educating women about breast cancer and promoting early detection practices amongst women in both the acute and community health care settings. However, nurses will have to be supported in this role, if this health-promoting endeavour is to become a reality.

Mairin O'Mahony is a College Lecturer and PhD student in the Catherine McAuley School of Nursing \& Midwifery, Brookfield Health Sciences Complex, under the supervision of : Professor Josephine Hegarty PhD, MSc, BSc, RNT, RGN, Acting Head of School of Nursing and Midwifery, Brookfield Health Sciences Complex, University College, Cork, Ireland and Professor Geraldine McCarthy, PhD, MSN, MEd, Dip Nursing, RNT, RGN, Acting Head of College of Medicine and Health, University College, Cork, Ireland. 